ECCOMAS

Proceedia
COMPDYN 2021

$8^{\text {th }}$ ECCOMAS Thematic Conference on Computational Methods in Structural Dynamics and Earthquake Engineering

M. Papadrakakis, M. Fragiadakis (eds.)

\title{
SEISMIC ACTION COMBINATION RULES FOR THE DESIGN OF AZIMUTH-INDEPENDENT STRUCTURES
}

\author{
Nikolaos Karaferis ${ }^{1}$, Dimitrios Vamvatsikos ${ }^{1}$ \\ ${ }^{1}$ National Technical University of Athens \\ 9, Iroon Polytechneiou Str., Zografou Campus, GR-15780, Athens, Greece \\ e-mail: \{nkaraferis, divamva\}@mail.ntua.gr
}

\begin{abstract}
The validity of the typical 100/30 combination rule for horizontal seismic action effects is investigated for the design of structures that are axially symmetric along the vertical direction. The 100/30 rule stipulates that one should combine 100\% of the seismic action in one principal direction (as estimated by the design spectrum) with $30 \%$ of the action in the other principal direction, and vice-versa. Having been derived for azimuth-dependent structures, having e.g., a rectangular plan, it takes advantage of the fact that the two horizontal components of ground motion are only partially correlated, with peaks that in general do not happen simultaneously, to reduce the overall design loads. On the contrary, vertical liquidstorage tanks, silos and chimneys are examples of azimuth-independent structures, which by virtue of their symmetry will always experience the worst-possible incidence angle of a ground motion. To quantify the effect of axisymmetry we employed a database of 150 records with three components of ground motion. The results show that an 106/106 combination rule, or more accurately a 1.12 amplification factor on the design spectrum in a single direction, rather than the 1.04 implied by the 100/30, is adequate to account for the effects of axisymmetry. Still, this value depends on the definition of the underlying design spectrum, and whether, e.g., the maximum, arbitrary or geometric mean component is employed, which should be accounted for in all calculations.
\end{abstract}

Keywords: Azimuth Independant Structures, Combination Rules, Horizontal Seismic Components, Structural Dynamics, Earthquake Engineering.

ISSN:2623-3347 C 2021 The Authors. Published by Eccomas Proceedia. Peer-review under responsibility of the organizing committee of COMPDYN 2021. doi: $10.7712 / 120121.8854 .18598$ 


\section{INTRODUCTION}

In seismic design, engineers address the structure's seismic capacity by applying an acceleration, and therefore a seismic action to the structure, which corresponds to the site where the structure is going to be located. Those values are provided from hazard maps and are specified in the design code [1-3]. In typical structures, it is also the case that two orthogonal horizontal axes are defined, corresponding to the principal directions of the structure, along which the ground motion and therefore the acceleration is applied. As is often the case in modal response spectra analysis (MRSA) or equivalent lateral force (ELF), the response of the structure is calculated separately by applying the design spectra per each of the principal axes. After the response of the structure has been successfully calculated in each, specific combination rules are applied to combine the results of both directions in a singular peak response for designing the structure [4]. Many combination rules and methods have been described in the literature [5] and have been adopted by different design codes, for example the combination rules described in Eurocode 8 [1], for seismic design, proposes the $100+30 \%$ rule [6] that suggests that for a specific response we consider the following cases:

$$
\begin{aligned}
& E_{E d x} "+" 0,30 E_{E d y} \\
& 0,30 E_{E d x} "+" E_{E d y}
\end{aligned}
$$

where the "+" symbol typically signifies a square-root-sum-of-square (SRSS) combination of the effects of the two horizontal components, rather than an actual addition.

This whole process has been chosen after investigations suggesting that with the assumptions above we can safely combine the responses of a building in its principal axes $\mathrm{X}$ and $\mathrm{Y}$, without being overly conservative. It would not be realistic to consider that in each direction the worst case values of acceleration would occur simultaneously [7]. This is of course an effective approach for the usual case of azimuth-dependent structures, but there is an obvious difference in case of structures that are fully symmetric with respect to a vertical axis and therefore have azimuth-independent properties, like cylindrical silos, spherical or vertical cylindrical liquid-storage tanks, circular pillars etc. In those structures the worst case scenario, considering the incidence angle of the ground motion, will always be in effect, as the structure has the same response and is equally vulnerable regardless of the axes defined.

In the following, a symmetrical three-dimensional elastic oscillator will be employed to examine its response after performing dynamic analysis using 150 pairs of strong ground motion records. The goal is to highlight the differences of such vertical axisymmetric structures when it comes to their response, ultimately calculating various amplification factors that allow combining the two horizontal components of motion when performing MRSA or ELF analysis for design purposes.

\section{GROUND MOTION ACCELERATION DEFINITIONS}

To properly clarify the process presented in the following paragraphs, in this segment some definitions [8] of how the ground motion acceleration parameters are usually defined in hazard maps provided by most design codes, are necessary. The following parameters represent the acceleration values calculated for a pair of records of the horizontal components derived for a specific earthquake:

- $\mathrm{Sa}_{\mathrm{RotD} 100}$ : The maximum acceleration value from all possible orientations of the records for a specific response period $\mathrm{T}$.

- $\mathrm{Sa}_{\mathrm{RotD} 50}$ : The median value from all possible orientations for a specific period $\mathrm{T}$. 
- $\mathrm{Sa}_{\mathrm{GMRotD100}}$ : The maximum value of the geometric mean of the horizontal components from all possible orientations of the paired records for period $\mathrm{T}$.

- $\mathrm{Sa}_{\mathrm{GMRotD50}}$ : The median value of the geometric mean of the horizontal components from all possible orientations for period $\mathrm{T}$.

- $\mathrm{Sa}_{\mathrm{arb}}$ : An "arbitrary" horizontal component of the ground motion for a specific period $\mathrm{T}$, as estimated by rotating the as-recorded components of the ground motion so that one coincides with a given axis

- $\mathrm{Sa}_{\mathrm{GM}}$ : Geometric Mean of as-recorded horizonal components for period T:

$$
\operatorname{Sa}_{\mathrm{GM}}(\mathrm{T})=\sqrt{ }\left[\operatorname{Sa}_{\mathrm{x}}(\mathrm{T}) \times \operatorname{Sa}_{\mathrm{y}}(\mathrm{T})\right]
$$

The above acceleration definitions are generally referring to acceleration values that are taking into account the incidence angle of the ground motion, meaning that for example for a value of $\mathrm{Sa}_{\mathrm{GMRotDXX}}$ the response of the structure is calculated by rotating the ground motion records in all angles (for example with a suitable step of $2^{\circ}$ ), and calculating the geometric mean value of the horizontal components, with the D50 or D100 value being chosen accordingly, in other words the median or maximum value could be adopted. In most codes the median values are adopted $\left(\mathrm{Sa}_{\mathrm{RotD} 50}, \mathrm{Sa}_{\mathrm{GMRotD50}}\right)$ taking into account that the principal axes of most structures, would not align to what the critical incidence angle of the ground motion would be, something though that as mentioned, is not true for azimuth independent structures. At this point it should be also clarified that in many cases in design codes the $\mathrm{Sa}_{\mathrm{Rot} 50}$ (periodindependent-rotation-angle measure) is adopted, but according to available literature [9, 10], the differences are insignificant between the RotDXX or RotIXX definitions.

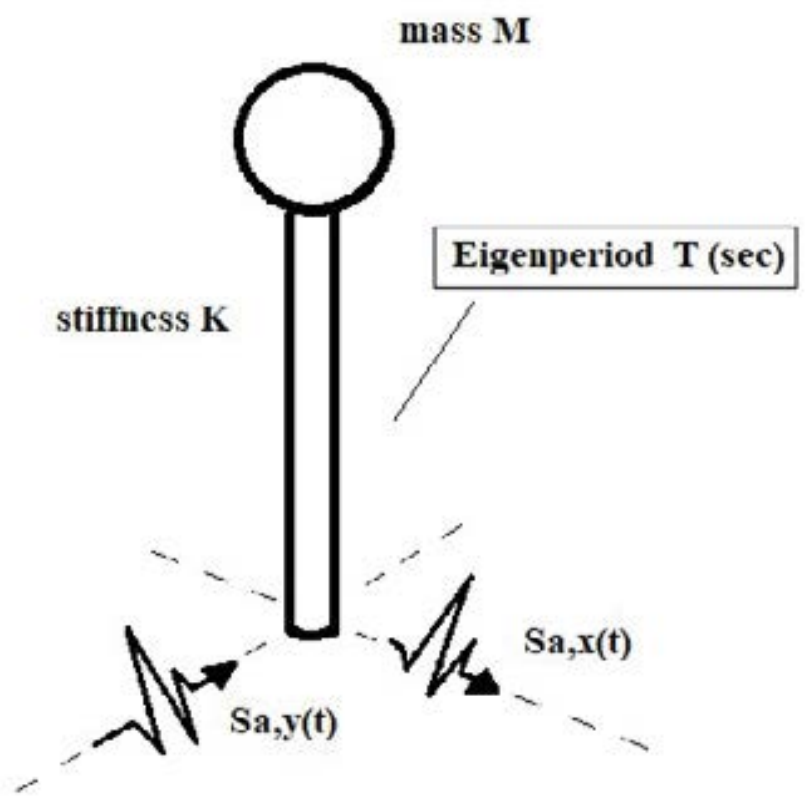

Figure 1: A perspective image of the 3 dimensional elastic and symmetrical oscillator's model, showing the common mass, stiffness and period in both horizontal axes. 


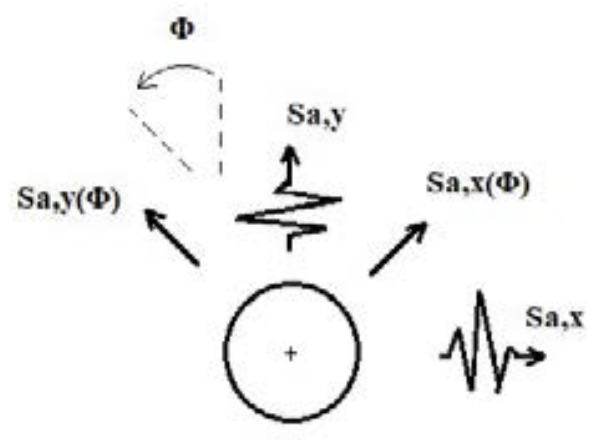

Figure 2: Incident angle rotation of horizontal ground motion components.

\section{OSCILLATOR MODEL AND DYNAMIC ANALYSIS PARAMETERS}

To calculate the acceleration values mentioned above, a three-dimensional elastic and symmetrical oscillator will be used, having the same dynamic parameters in both its major directions $\mathrm{X}$ and $\mathrm{Y}$. Using all the aforementioned 150 pairs of ground motion records, from earthquakes of various characteristics, dynamic analysis is performed in each case of record pairs, rotating the ground motion accelerations with an angle step of $2^{\circ}$ through a complete circle, essentially altering in each case the incidence angle of the ground motion accelerations.

Through this process and by performing those calculations for all possible incidence angles starting from $0^{\circ}$ to $180^{\circ}$ (with the previously mentioned $2^{\circ}$ step), the accelerations mentioned in the previous paragraph are easily defined, providing a range of responses that can be used to convert the GMRotD50 and RotD50 values in the more suitable D100 cases, that as mentioned before are more reasonable for axisymmetric structures. In Figures 3-4 the results for a specific case of an oscillator with an eigenperiod of $\mathrm{T}=1.0 \mathrm{sec}$ are presented to put into perspective the process in a more practical way.

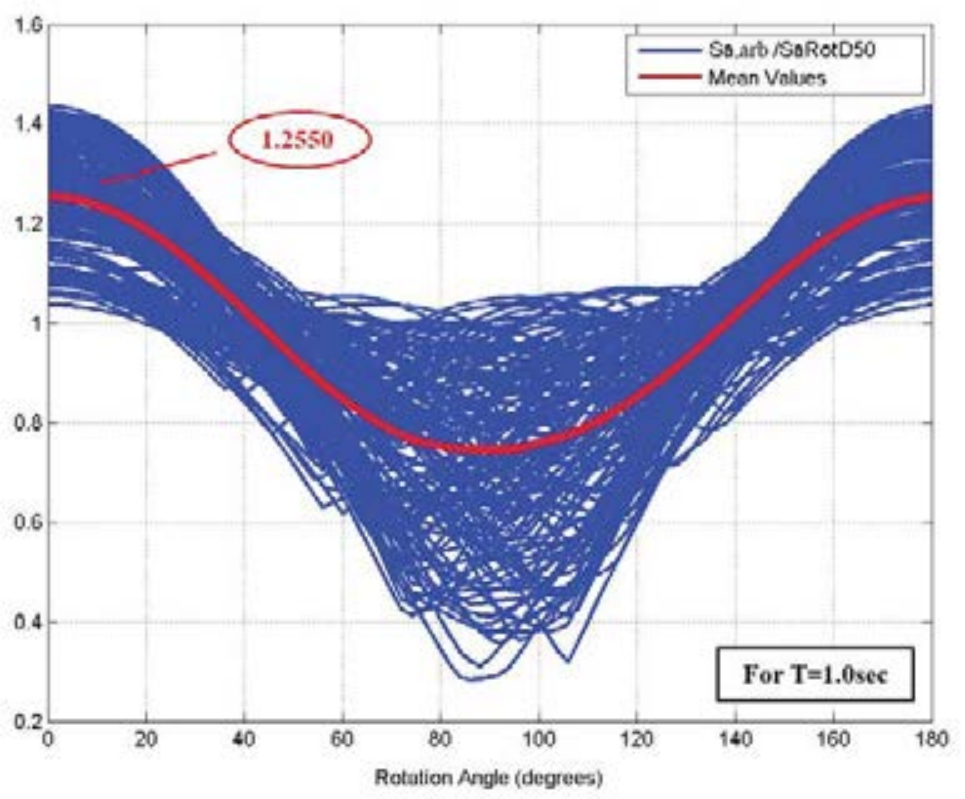

Figure 3 : Results for each record pair, with diagrams for $\mathrm{Sa}_{\mathrm{arb}}(\theta) / \mathrm{Sa}_{\mathrm{RotD} 50}$ and the mean value for each rotation angle (arbitrary case). 


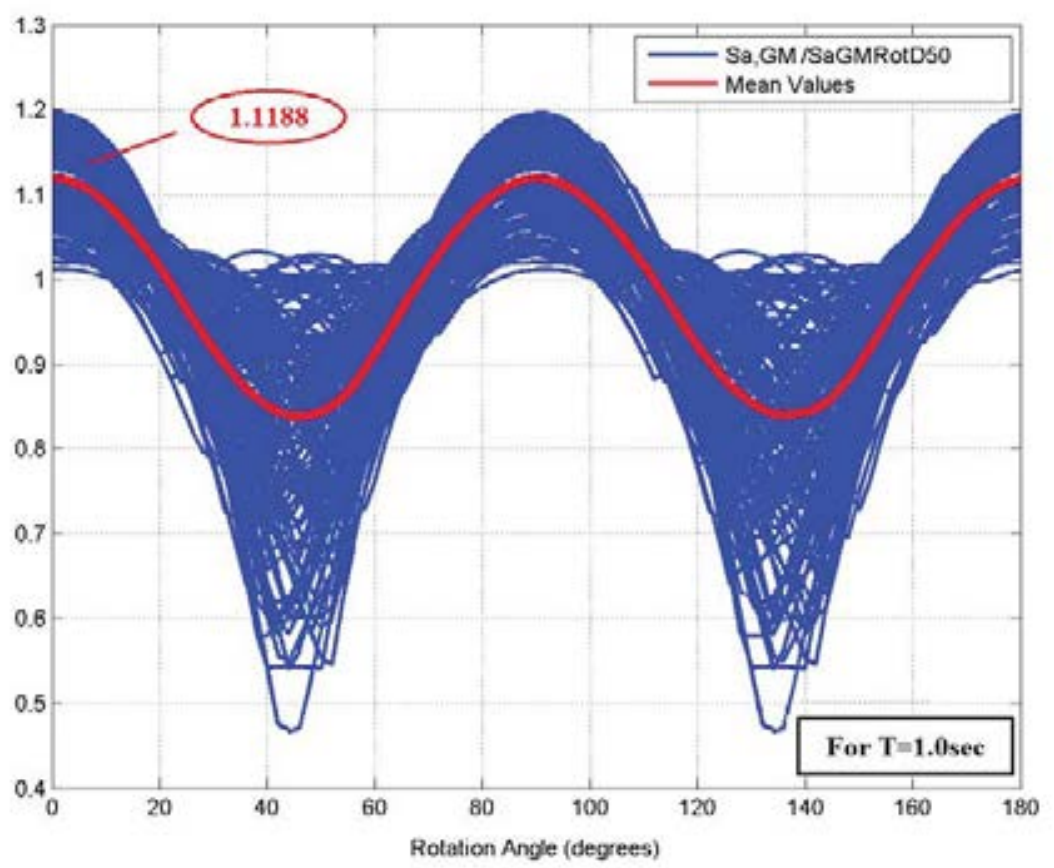

Figure 4 : Results for each record pair, with diagrams for $\operatorname{Sa}_{\mathrm{GM}}(\theta) / \operatorname{Sa}_{\mathrm{GMRotD} 50}$ and the mean value for each rotation angle (geomean case).

It is very important to mention that to better identify the output of the results, each diagram for each record was plotted starting from the incidence angle where the maximum acceleration was found. Of course the results were also divided with the equivalent $\mathrm{Sa}_{\mathrm{RotD} 50}$ and $\mathrm{Sa}_{\mathrm{G}-}$ MRotD50 values accordingly, for the results, concerning each pair of records, to be comparable to each other (in the second case where the geometric mean values are examined, after the $90^{\circ}$ angle the diagrams repeat themselves).

\section{RESULTS}

Using the process mentioned above for a wide spectrum of eigenperiods $\mathrm{T}(\mathrm{sec})$ for the oscillator described, and by identifying the mean value for each of the factors, appearing in Figure 5, for every $\mathrm{T}$, taking into account all 150 records, the more condensed diagrams were created. They of course provide a wider perspective of how the different definitions of ground motion acceleration relate to one another, examining not only the obvious cases of $\mathrm{Sa}$ -

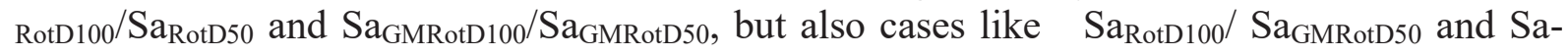
RotD50/Sa $/$ GMRotD50, in case a conversion of that kind could be useful.

According to the results of this investigation, it is obvious that if the peak horizontal acceleration value of a common structure according to the $100+30 \%$ rule, and combining the two horizontal components with the SRSS rule would be $1.044 \cdot \mathrm{a}_{\mathrm{g}}$. In a case where the hazard maps proposed are set up using the $\mathrm{Sa}_{\mathrm{GMRotD50}}$ definition, which is expected to be the case of Eurocode, an amplification factor of $1.117 \cdot \mathrm{a}_{\mathrm{g}}$ should be employed. A value between 1.20 $1.25 \cdot \mathrm{a}_{\mathrm{g}}$ is proper in case of a design spectrum definition based on $\mathrm{Sa}_{\mathrm{RotD} 50}$. Those are significant differences that certainly should not be disregarded when axisymetric structures are examined, as this could lead to non conservative results. At this point a reference of similar works can verify some of the results above especially in cases of the $\mathrm{Sa}_{\mathrm{RotD} 100} / \mathrm{Sa}_{\mathrm{RotD} 50}$ diagrams for example, as similar results have appeared in the literature [9, 11]. 


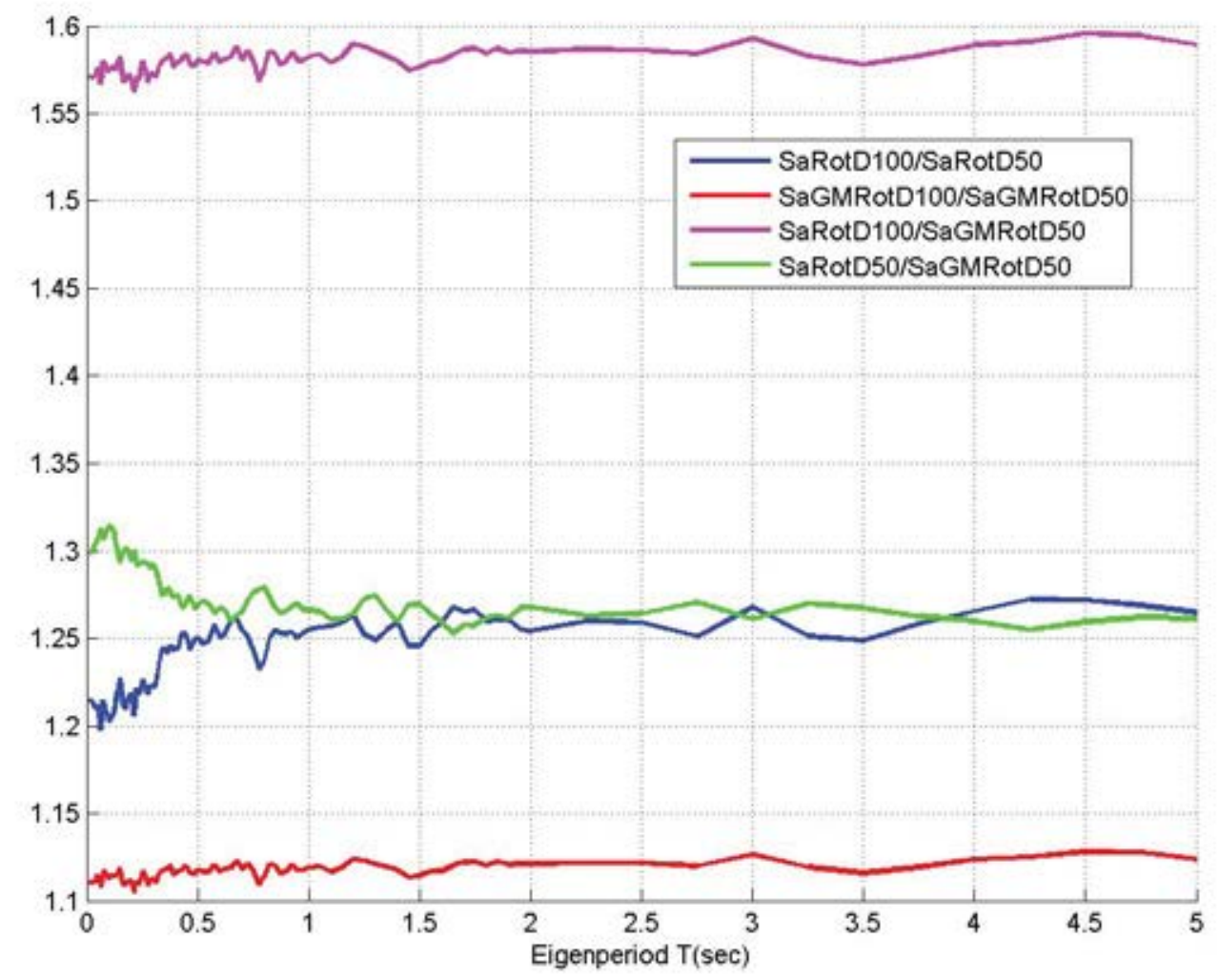

Figure 5 : Aggregated results for various amplification factors for various eigenperiods $\mathrm{T}$.

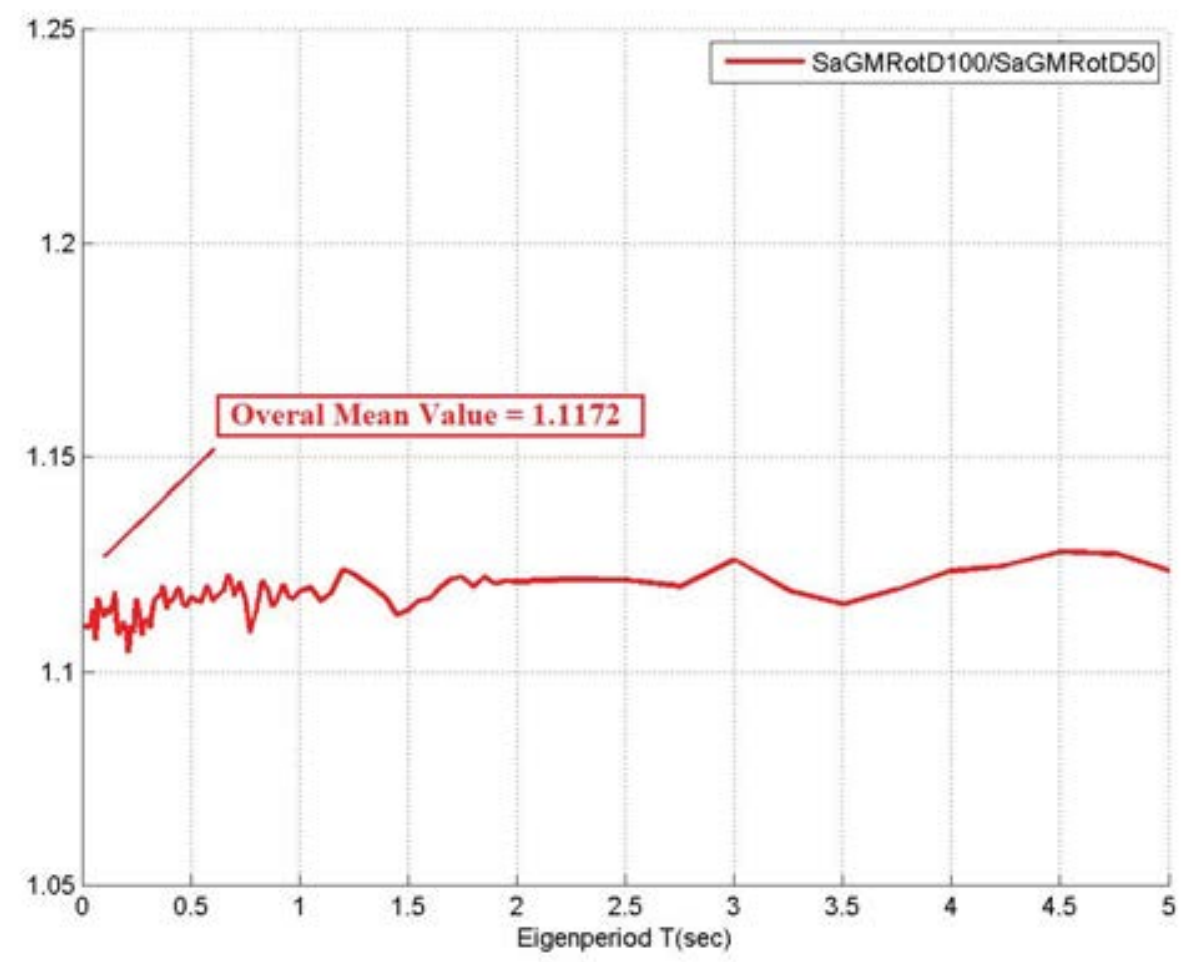

Figure 6 : Aggregated results for the $\mathrm{Sa}_{\mathrm{GMRotD} 100} / \mathrm{Sa}_{\mathrm{GMRotD} 50}$ conversion factor for various eigenperiods $\mathrm{T}$. 


\section{CONCLUSIONS}

To conclude, the perspective of this article is to propose specific amplification factors in cases of azimuth-independent structures. Their symmetry suggests that the seismic accelerations that would affect them would act upon them in maximum effect, as the incidence angle of the ground motion will affect the structure regardless of the axis X and Y arbitrarily defined in the design process, always subjecting the structure in the peak acceleration produced by the earthquake. That is why we propose amplification factors for the horizontal acceleration component used in design, of 1.12 in a case of an acceleration definition of $\mathrm{Sa}_{\text {GMRotD50 }}$ in the hazard maps and an amplification factor of 1.25 for the Sa $\mathrm{RotD50}_{0}$ case accordingly. Specifically for Eurocode 8 the authors propose the 1.12 case, as an amplification factor for the design spectrum accelerations applied in a single direction to simultaneously account for the combined effect of both horizontal components in axisymetric structures.

\section{AKNOWLEDGEMENT}

This research has been co-financed by the European Regional Development Fund of the European Union and Greek national funds through the Operational Program Competitiveness, Entrepreneurship and Innovation, under the call RESEARCH - CREATE - INNOVATE (project code: T1EDK-00956), project: "ARCHYTAS: Archetypal telemetry and decision support system for the protection of monumental structures".

\section{REFERENCES}

[1] CEN, Design of structures for earthquake resistance - Part 1: General rules, seismic actions and rules for buildings, EN1998-1, European Committee for Standardisation, Brussels, 2005.

[2] T.D. Ancheta, R.B. Darragh, J.P. Stewart, E. Seyhan, W.J. Silva, B.S.J. Chiou, K.E. Wooddell, R.W. Graves, A.R. Kottke, D.M. Boore, T. Kishida, J.L. Donahue, NGAWest2 database, Earthquake Spectra, 30, 989-1005, 2014.

[3] J.W. Baker, C.A. Cornell, Which spectral acceleration are you using?, Earthquake Spectra, 22, 293-312, 2006.

[4] O.A. Lopez, A.K. Chopra, J.J. Hernandez, Evaluation of combination rules for maximum response calculation in multi component seismic analysis, Earthquake Engineering \& Structural Dynamics, 30, 1379-1398, 2001.

[5] C. Menun, A. Der Kiureghian, A replacement for the 30\%, 40\%, and SRSS rules for multicomponent seismic analysis, Earthquake Spectra, 14, 153-163, 1998.

[6] E. Rosenblueth, H. Contreras, Approximate design for multicomponent earthquakes, Journal of the Engineering Mechanics Division, 103, 881-893, 1977.

[7] J.P. Stewart, N.A. Abrahamson, G.M. Atkinson, J.W. Baker, D.M. Boore, Y. Bozorgnia, K.W. Campbell, C.D. Comartin, I.M. Idriss, M. Lew, M. Mehrain, J.P. Moehle, F. Naeim, T.A. Sabol, Representation of bidirectional ground motions for design spectra in building codes, Earthquake Spectra, 27, 927-93, 2011. 
[8] D.M. Boore, J. Watson-Lamprey, N.A. Abrahamson, Orientation-independent measures of ground motion, Bulletin of the seismological Society of America, 96, 1502-1511, 2006.

[9] D.M. Boore, Orientation-independent, nongeometric-mean measures of seismic intensity from two horizontal components of motion, Bulletin of the Seismological Society of America, 100, 1830-1835, 2010.

[10] K. Beyer, J.J. Bommer, Selection and scaling of real accelerograms for bi-directional loading: a review of current practice and code provisions, Journal of earthquake engineering, 11, 13-45, 2007.

[11] S.K. Shahi, J.W. Baker, NGA-West2 models for ground motion directionality, Earthquake Spectra, 30, 1285-1300, 2014. 